\title{
L'analyse logique comme outil de soutien à l'application des connaissances dans le domaine psychosocial
}

\author{
Anne-Marie Ouimet et Daphné Morin \\ Centre de recherche et d'expertise sur la déficience intellectuelle et le \\ trouble du spectre de l'autisme, Centre intégré universitaire de santé \\ et de services sociaux du Centre-Sud-de-l'île-de-Montréal
}

Résumé : Les organisations publiques offrant des services sociaux, notamment en déficience intellectuelle, déploient des efforts depuis une quinzaine d'années pour rehausser leurs pratiques par une plus grande adéquation avec les connaissances scientifiques. L'analyse logique, une approche ancrée dans lévaluation théorique d'intervention, constitue une avenue prometteuse pour l'application des connaissances en milieux de pratique. À travers la description d'une analyse logique d'un programme de développement des habiletés sociales, cet article souligne ses retombées, soit le déploiement d'une pratique cohérente avec les connaissances scientifiques actuelles ainsi que la valorisation et la mise à profit des connaissances issues de l'expérience des conceptrices du programme.

Mots clés : analyse logique, application des connaissances, évaluation théorique

\begin{abstract}
For fifteen years, social services organizations, such as those working with people with intellectual disabilities, have been deploying numerous efforts to enhance their practices by making them more consistent with scientific knowledge. Logical analysis, a theory-based evaluation approach, is a promising avenue for knowledge translation in practice settings. By describing the experience of applying that particular analysis framework to a social skill program, this article sheds light on the benefits of using that approach, such as implementing a practice consistent with current scientific knowledge and valuing the program designers' experiencebased knowledge.
\end{abstract}

Keywords: logical analysis, knowledge application, theoretical evaluation.

\section{INTRODUCTION}

Depuis leur intégration dans le réseau de la santé et des services sociaux, et de manière plus intensive avec l'adoption d'une politique intitulée De l'intégration

Correspondance à l'auteur : Daphné Morin, Centre intégré universitaire de santé et de services sociaux du Centre-Sud-de-l'Île-de-Montréal (CIUSSS-CSIM), 1001, De Maisonneuve Est, Montréal, Québec, Canada H2L 4R5; courriel : daphne.morin.ccsmtl@ssss.gouv.qc.ca 
sociale à la participation sociale. Politique de soutien aux personnes présentant une déficience intellectuelle, à leur famille et aux autres proches (Ministère de la Santé et des Services sociaux, 2001), les organisations offrant des services sociaux d'adaptation et de réadaptation en déficience intellectuelle (DI) ont été appelées à se spécialiser et à se professionnaliser, entraînant ainsi des exigences de pratiques basées sur des données probantes. Ces organisations tentent d'adapter leurs pratiques à ces exigences, mais sont confrontées à des défis importants : accessibilité des services, spécialisation des pratiques, transformation des profils de populations à desservir, nouveaux rôles sociaux des personnes présentant une DI, etc. Ces organisations s'interrogent, notamment, sur les manières de transformer leurs pratiques afin quelles reposent sur des connaissances scientifiques, mais sans balayer du revers de la main les pratiques fondatrices de la discipline, tout en valorisant les savoirs issus de l'expérience. Les collaborations étroites entre ces organisations et les milieux académiques sont en général plus récentes, ce qui explique, en partie du moins, l'état actuel de la culture de recherche dans ces organisations. De plus, à ce jour, l'état des connaissances quant aux stratégies à privilégier par ces organisations pour favoriser l'application des connaissances dans leur milieu demeure limité (Chaire CJM-IU-UQAM, s.d.).

Cet article vise à démontrer qu'une méthode novatrice d'évaluation, l'analyse logique, peut constituer un outil prometteur et utile pour soutenir l'application de connaissances à des interventions du domaine psychosocial. Cette méthode sera présentée brièvement et ensuite explorée à travers un retour sur l'expérience de l'analyse logique d'ADOPRO, un programme de développement d'habiletés sociales adapté à la déficience intellectuelle (CRDITED de Montréal, 2015).

\section{L'ANALYSE LOGIQUE EN SOUTIEN À L'APPLICATION DES CONNAISSANCES}

\section{L'évaluation au profit de l'application des connaissances}

L'Organisation mondiale de la santé définit l'application des connaissances (AC) comme :

... l'échange, la synthèse et la communication efficace de résultats fiables et pertinents issus de la recherche. Laccent est mis sur la promotion de l'interaction entre les producteurs et les utilisateurs de la recherche, lélimination des obstacles à l'utilisation de la recherche et l'adaptation de l'information en fonction des divers publics cibles, de façon à élargir la portée des interventions efficaces... (World Health Organization, 2004, p. 140, traduction libre).

Ainsi, l'AC dépasse largement la dissémination de résultats de recherche. Elle vise non seulement leur appropriation par les milieux de pratique, mais également une appropriation des connaissances et des besoins des milieux de pratique par les chercheurs. Elle présuppose donc des processus itératifs de communication et de partage entre les différentes parties. Il existe d'autres formes de processus 
conceptualisant le mouvement entre la recherche et l'action, telles que le transfert des connaissances, la dissémination, la valorisation des connaissances, l'utilisation des connaissances, etc. L'AC se distingue par 1) les interactions et l'engagement entre les différents acteurs concernés (ex. : utilisateurs, producteurs); ainsi que par 2) son caractère itératif et multidimensionnel (Davison, 2009). Finalement, son succès repose sur la qualité des interactions qu'elle a engendrée et sur l'utilisation des connaissances ciblées par les milieux, par exemple, lorsque celles-ci ont été utilisées afin d'aider la création et l'amélioration d'interventions (Davison, 2009).

Bien que le domaine de l'évaluation s'intéresse à la question de l'utilisation des connaissances issues d'évaluations depuis plusieurs années (Blake et Ottoson, 2009), la question de l'utilisation de l'évaluation comme stratégie de soutien à l'AC n'a été que très peu traitée. Donnelly, Letts, Klinger et Shulha (2014) ont récemment développé une approche d'évaluation fondée sur l'AC qui repose sur l'utilisation des connaissances et sur leur mise en valeur à travers les différentes étapes du processus d'évaluation. Mise à l'épreuve dans le cadre d'un projet d'évaluation où l'évaluateur principal jouait aussi un rôle de courtier de connaissances, cette expérience a favorisé la prise en compte de connaissances scientifiques lors de la conception du programme, tout en valorisant les connaissances issues de la pratique (Donnelly et coll., 2014).

\section{Description de la méthode d'analyse logique}

Issue des méthodes d'évaluation théorique de programmes, l'analyse logique est un des types d'analyse du continuum de la recherche évaluative qui s'intéresse à la cohérence de l'intervention et qui permet de porter un jugement sur le bien-fondé théorique d'un programme à partir de connaissances scientifiques ou de l'avis d'experts (Champagne, Brousselle, Contandriopoulos et Hartz, 2009). En analysant l'applicabilité et la validité des liens causaux entre les composantes d'une intervention, elle permet d'anticiper le potentiel des actions prévues sur l'atteinte des objectifs visés (Brousselle et Champagne, 2011). Plus spécifiquement, l'analyse logique vise à porter un jugement sur la nature de l'intervention (cohérence entre les hypothèses des causes du problème et celles de l'intervention - modèle causal, modèle logique théorique) et sur ses modalités de mise en application (justesse des moyens retenus - modèle logique opérationnel) (Champagne et coll., 2009) ${ }^{1}$.

Bien que des postulats aujourd'hui associés à l'analyse logique aient été identifiés dans la littérature en évaluation depuis de nombreuses années (caractère évaluable de l'intervention, bien fondé théorique comme critère de jugement sommatif et comme déterminant de l'efficacité), la mise en application de ce type d'analyse n'a été que peu documentée (Brousselle et Champagne, 2011; Tremblay, Brousselle, Richard et Beaudet, 2013). Selon les rares expériences rapportées, cette méthode favoriserait le développement de programmes basés sur des théories d'intervention scientifiquement valides, tout en exigeant peu de ressources humaines et financières (Brousselle et Champagne, 2011; Tremblay et coll., 2013). 
La prochaine section illustrera l'application de cette méthode pour l'évaluation d'un programme de développement des habiletés sociales en DI par une équipe de chercheuses rattachées à une direction de recherche en milieu de pratique dans lequel le programme a été élaboré.

\section{PRÉSENTATION DE L'EXPÉRIENCE D'ANALYSE LOGIQUE D'ADOPRO}

Le projet d'analyse logique d'ADOPRO est issu d'une volonté d'un centre de réadaptation en DI et en trouble du spectre de l'autisme de diffuser ce programme conçu par des intervenantes et des professionnelles. Inspiré d'autres programmes similaires, il a été développé afin de combler les limites de ces derniers, puis bonifié au fil des ans à partir de l'expérience des conceptrices qui l'ont appliqué pendant six années auprès d'une clientèle d'adolescents présentant une DI. Des présentations du programme dans le cadre de colloques ont suscité la demande et amené la direction de l'établissement à formaliser sa publication. Engagée dans un processus de développement de sa mission universitaire, la direction souhaitait au préalable s'assurer de la validité scientifique du programme qui, à première vue, paraissait essentiellement fondé sur des savoirs tacites, c'est-à-dire des savoirs implicites ou dont on ne connait pas la source. C'est dans cette perspective que la direction de recherche de cet établissement, en tant que direction de soutien à la valorisation des connaissances dans l'organisation, a proposé la réalisation d'une analyse logique du programme ADOPRO.

Cette analyse logique s'est déroulée en étroite collaboration avec deux des conceptrices du programme qui, en plus d'agir en tant qu'informatrices, apportaient des ajustements au programme en cours de route. Elle s'est déroulée en trois grandes étapes : 1) la modélisation du programme; 2) l'analyse de l'adéquation des connaissances issues d'une revue de la littérature scientifique avec les composantes du programme; et 3 ) une consultation d'experts.

\section{Étape 1 : Modélisation du programme}

Cette étape visait, d'une part, à rendre plus explicites les composantes et les objectifs du programme. En effet, il comportait une multitude d'approches d'enseignement et de cibles d'apprentissage difficiles à concevoir dans leur globalité et leur finalité. D'autre part, ces travaux allaient permettre de mettre en lumière les raisons et les connaissances ayant motivé l'intégration de ses différentes composantes et le bien-fondé de ses objectifs.

Ainsi, des rencontres d'échange avec les deux conceptrices du programme, la consultation des documents du programme, puis une recension d'articles scientifiques sur les habiletés sociales ont été effectuées. Ensuite, un schéma représentant les causes du problème, ou arbre causal, associées au déficit sur le plan des habiletés sociales chez les adolescents ayant une DI, a été construit à partir des connaissances des conceptrices et des connaissances issues des articles scientifiques consultés. Le modèle logique du programme a, quant à lui, été élaboré à partir des documents du 
programme et des consultations des conceptrices. Finalement, une schématisation des cibles d'apprentissage a été développée afin de clarifier les contenus abordés dans le programme. Ces modèles ont ensuite été présentés aux conceptrices à des fins de validation. Celles-ci ont pu y refléter leur compréhension de la problématique et des composantes du programme, tout en y apportant des éléments de justification à partir de leurs connaissances professionnelles et expérientielles.

Ensuite, les évaluatrices et les conceptrices ont procédé à une première validation de la modélisation du programme par un exercice de mise en relation de l'arbre causal, du modèle logique et de la schématisation des cibles d'apprentissage. Cette phase d'analyse a entraîné une restructuration des objectifs et des cibles d'apprentissage du programme, sans en changer profondément la teneur (ex. : des cibles d'apprentissage de la modélisation étaient formulées, dans les versions initiales, en objectifs généraux ou spécifiques du programme).

\section{Étape 2 : Analyse de l'adéquation des connaissances scientifiques avec les composantes du programme}

Cette deuxième étape visait principalement à s'assurer que les différentes composantes du programme et ses objectifs étaient appuyés par les connaissances scientifiques actuelles.

Pour ce faire, une revue de la littérature scientifique portant sur des programmes d'habiletés sociales a d'abord été effectuée. Cette revue non systématique a été réalisée au moyen d'une consultation itérative de bases de données bibliographiques et de plateformes de recherche Web. À partir des articles retenus en raison de leur pertinence, une mise en parallèle de leurs contenus avec les composantes d'ADOPRO a été réalisée. Lorsque des différences majeures étaient identifiées (ex. : approches privilégiées par d'autres programmes, recommandations issues d'évaluations), celles-ci étaient discutées avec les conceptrices du programme ADOPRO, entraînant une analyse plus approfondie des choix théoriques (ex. : nouvelles recherches de littérature) et leur clarification sur la base de la mise en commun des savoirs.

Ainsi, ces discussions ont mené parfois à une prise de position différente de celle émergeant des écrits scientifiques. Par exemple, la majorité des programmes recensés s'inscrivaient dans une approche de type cognitivo-comportemental, qui sous-tend, notamment de développer une voix intérieure modulant les comportements. Or, selon l'expérience des conceptrices, l'utilisation de ce type d'approche pour le développement des habiletés sociales auprès d'adolescents ayant une DI est inefficace, car trop abstrait, constat qui est d'ailleurs ressorti lors de la consultation d'experts (voir étape 3). Le programme ADOPRO s'inscrit plutôt dans une approche de type behavioral qui mise sur l'utilisation systématique de renforçateurs positifs. Lanalyse logique a ainsi permis de mettre en lumière la valeur des connaissances issues de la pratique clinique lorsqu'elles sont basées sur un argumentaire solide. Aussi, cet exercice et ce choix théorique suggèrent de porter une attention à cet aspect du modèle lors de phases subséquentes d'évaluation dans le but de renforcer la robustesse du bien-fondé théorique du programme. 


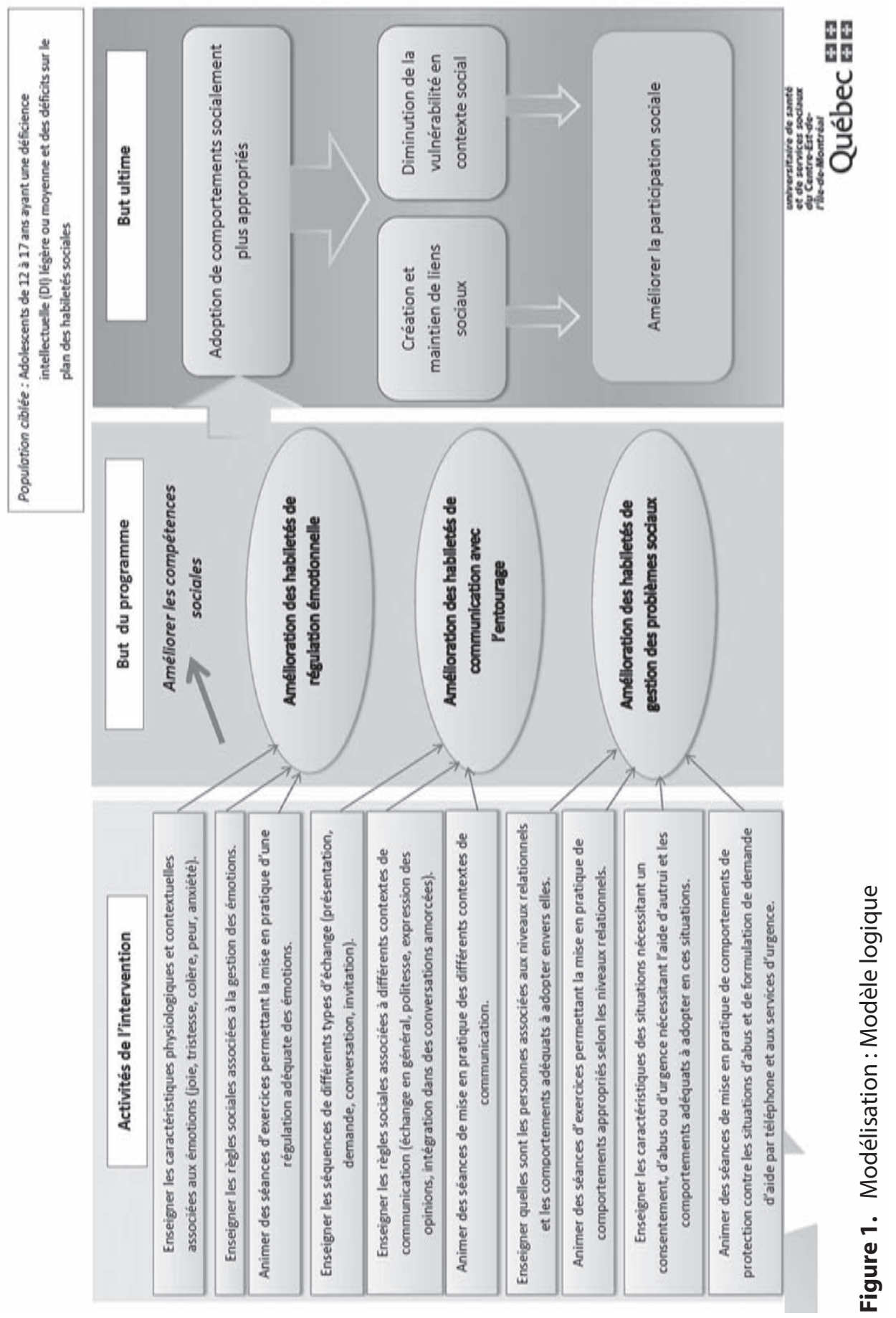




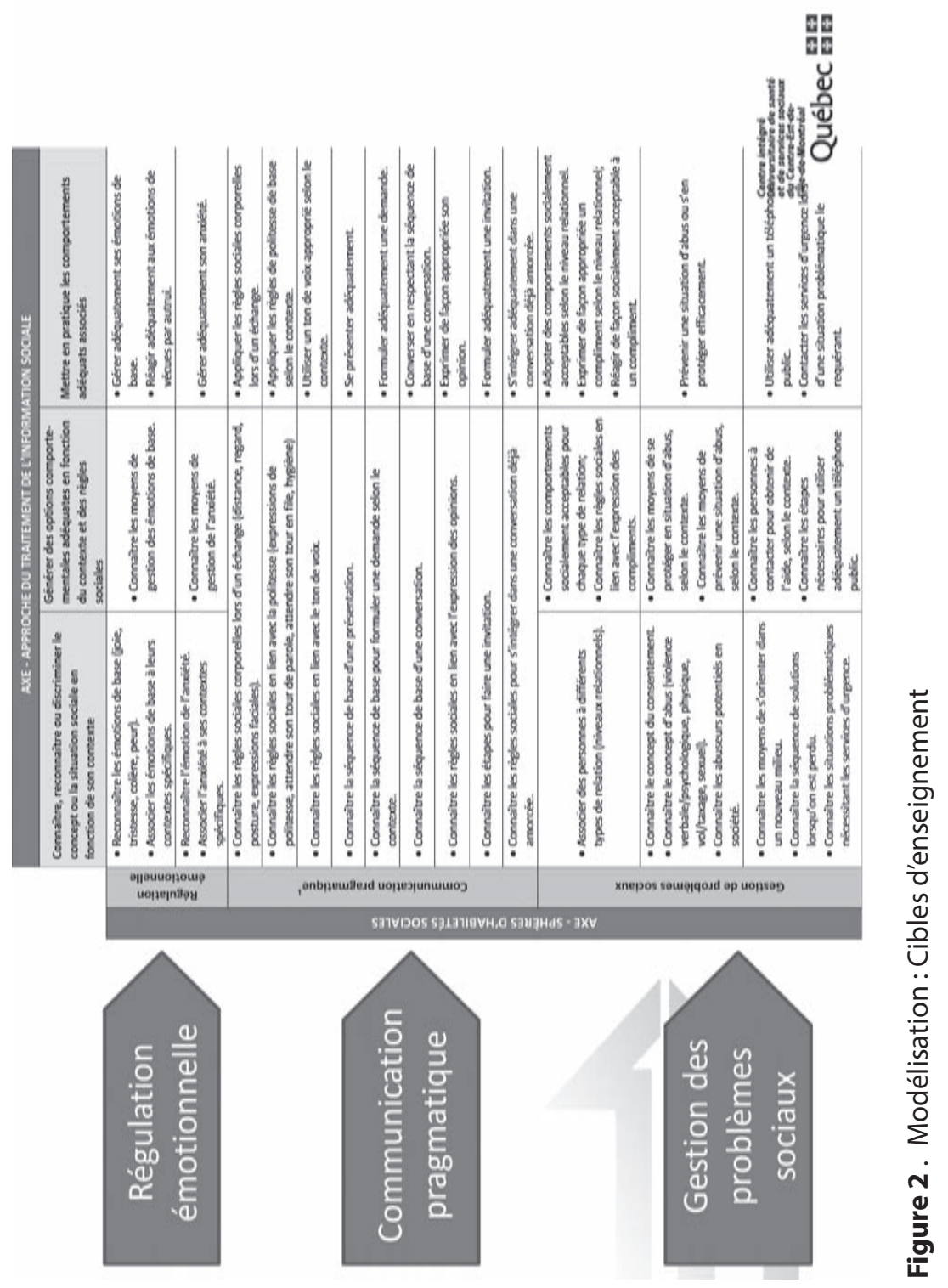




\section{Étape 3 : Consultation d'experts}

Cette dernière étape poursuivait deux objectifs : 1) permettre une triangulation des connaissances issues des savoirs d'experts avec celles issues de la littérature scientifique et de l'expérience des conceptrices; et 2) juger de la pertinence des moyens et des ressources utilisés dans le programme.

Pour la triangulation des connaissances, les évaluatrices, en tant que partie prenante du projet de publication du programme, ont d'abord elles-mêmes rédigé un document décrivant les fondements théoriques du programme à partir des informations recensées dans le cadre des deux premières étapes. Ce document allait permettre aux experts de se prononcer sur la capacité théorique du programme d'atteindre ses objectifs. Ces derniers ont été recrutés en fonction de leur expertise portant sur le développement de programmes en DI ou sur le développement des habiletés sociales. Ils ont formulé différents commentaires qui ont, par exemple, permis d'approfondir la réflexion quant à l'importance du réseau social des adolescents sur leurs habiletés sociales. Ce constat a entraîné l'intégration d'activités visant spécifiquement les partenaires scolaires et les parents des participants. Leurs commentaires ont également entraîné une justification plus approfondie de certains aspects du programme, notamment de l'approche théorique de type behavioral sur laquelle il s'appuie ou sur ses retombées possibles auprès d'adolescents ayant une DI moyenne.

Pour juger de la pertinence des moyens et des ressources du programme, une version révisée du Guide des animateurs du programme a été soumise aux experts. Harmonisé à la lumière de l'ensemble des modifications apportées au programme en cours de route, ce guide illustrait la logique opérationnelle du programme, soit les ressources nécessaires, les activités d'implantation, les cibles et les approches d'enseignement et les méthodes d'évaluation. D’autres documents, tels que des plans de cours et des grilles dévaluation, leur ont également été soumis. Consultés au moyen d'un canevas de questions ouvertes, les experts ont estimé que le programme révisé était théoriquement bien-fondé. Ils ont également formulé des recommandations de clarifications et de modifications mineures qui ont été prises en compte.

\section{DISCUSSION}

En plus de produire une validation du bien-fondé théorique du programme, cette analyse logique d'ADOPRO a permis d'apporter de multiples clarifications et bonifications au programme. Sa modélisation, en plus den rendre plus explicites les fondements et les composantes, a engendré un nouveau regard sur le concept d'habiletés sociales et a permis une meilleure définition des sphères d'habiletés sur lesquelles il importe d'agir auprès de cette clientèle. La deuxième étape, soit l'analyse de l'adéquation des connaissances issues d'une revue de la littérature scientifique avec les composantes du programme, a permis une valorisation d'aspects du programme fondés sur l'expérience des conceptrices. En effet, certains aspects qui rencontraient une certaine résistance organisationnelle, telle que la longue durée du programme ou encore la dernière session se déroulant dans le cadre d'une activité 
communautaire, se sont avérés être identifiés dans les écrits scientifiques comme des éléments favorables à l'efficacité de tels programmes auprès de cette clientèle. Finalement, les consultations d'experts ont constitué une valeur ajoutée au programme qui a pu être bonifié, en plus d'assurer la prise en compte d'un plus large éventail de connaissances qui auraient pu être échappées lors des revues de la littérature.

Lanalyse logique du programme ADOPRO représente un exemple concret du caractère prometteur de l'utilisation de cette méthode pour l'application des connaissances aux pratiques d'intervention du domaine psychosocial. En effet, elle s'inscrit dans une approche d'AC dans la mesure où elle contribue à favoriser des interactions soutenues entre des porteurs de différentes formes de savoirs et supporte la création et l'amélioration d'interventions (Davison, 2009). Dans le cas d'ADOPRO, elle a également permis de sensibiliser les conceptrices à l'importance de la prise en compte des connaissances scientifiques lors du développement de programme. Enfin, elle contribue à réduire l'incertitude quant à la contribution du programme, grâce à l'application des meilleures connaissances à une démarche évaluative essentielle (Mayne, 2001).

\section{Facteurs ayant contribué au succès de la démarche}

D’abord, en cohérence avec la démarche associée à l'AC (Davison, 2009), les conceptrices ont été impliquées de manière significative à chaque étape du processus d'évaluation. Appelées à réagir et à se positionner face à des constats issus des écrits scientifiques, ces dernières ont pu non seulement se les approprier, mais aussi se positionner lorsque leur expérience sur le terrain amenait une perspective différente. L'importance de cette contribution est également mise de l'avant par d’autres évaluateurs selon qui elle maximiserait l'appropriation des résultats de lévaluation et permettrait la valorisation d'autres formes de connaissances (ex. : expérientielles, professionnelles) que celles issues des connaissances scientifiques (Brousselle et Champagne, 2011; Tremblay et coll., 2013).

Tout comme pour l'équipe de Donnelly et coll. (2014) lors de leur mise à l'essai de l'approche d'évaluation fondée sur l'AC, l’engagement significatif des évaluatrices dans le façonnage du programme, notamment dans la rédaction d'un document détaillant ses fondements théoriques et ce qui allait devenir le Manuel du programme d'ADOPRO, constitue une autre condition du succès de la démarche et de ses retombées. Bien que ce niveau d'engagement puisse sembler outrepasser le rôle de l'évaluateur, il a favorisé l'utilisation concrète des connaissances au profit du programme. Ainsi, dans le cas d'ADOPRO, les connaissances, se trouvant intégrées à même la structure du programme, seront appliquées par les intervenants qui l'utiliseront. Cet engagement de léquipe dévaluatrices a été possible en raison de leur double mandat d'évaluation et de valorisation des connaissances au sein de leur organisation. De plus, étant donnée la disponibilité limitée des conceptrices en raison de leur mandat clinique, il savérait plus réaliste de confier ce mandat de rédaction aux évaluatrices qui avaient pu acquérir une bonne maitrise des contenus dans le cadre du projet.

Finalement, tout au long du processus d'évaluation, les conceptrices ont apporté des ajustements aux différents documents du programme. Si ces ajustements 
ont parfois dû être révisés en cours de processus puisque de nouvelles connaissances émergeaient, ce travail a néanmoins permis d'assurer une prise en compte concrète et systématique des constats issus de l'analyse.

\section{Nouvelle approche en analyse logique}

Depuis la réalisation de l'analyse logique du programme ADOPRO, la méthode s'est raffinée. Après avoir modélisé l'intervention ciblée, il est maintenant proposé de concevoir un modèle conceptuel des composantes essentielles nécessaires à l'atteinte des objectifs identifiés dans les écrits scientifiques ou par des experts, puis de comparer l'adéquation des deux modèles (Brousselle et Champagne, 2011). Dans le cadre du projet ADOPRO, la conception d'un tel modèle conceptuel aurait probablement facilité le processus d'évaluation. La démarche utilisée dans le cadre du projet ADOPRO a toutefois favorisé une appropriation graduelle et plus approfondie des connaissances scientifiques par les conceptrices et a conduit à des ajustements du programme sur une base continue en raison de leur participation au processus d'évaluation. De plus, en privilégiant une mixité des approches d'analyse, par des consultations itératives de la littérature et des experts, à une démarche segmentée en étapes indépendantes et successives, la démarche utilisée a permis une triangulation des résultats et a ainsi renforcé leur validité.

\section{CONCLUSION}

Lanalyse logique de programmes d'intervention psychosociale constitue une approche prometteuse de soutien à l'AC dans des milieux de pratique, particulièrement pour des acteurs ou des équipes ayant la responsabilité de la valorisation des connaissances au sein d’organisations. Elle permet d'amener une réflexion sur la nature des connaissances à la base d'une intervention et une mise à profit concrète et utile des connaissances scientifiques. Dans sa perspective évaluative, elle permet d'estimer l'évaluabilité d'une intervention en s'assurant que cette dernière repose sur des fondements théoriques valides (plausibilité des hypothèses, adéquation entre objectifs et moyens). Finalement, elle facilite la mise en œuvre d'évaluations d'efficacité ou d'implantation grâce à l'éclaircissement des composantes et des objectifs d'une intervention. Ainsi, en l'absence de guides ou d'orientations destinés aux organisations souhaitant intégrer et appliquer davantage de connaissances scientifiques à leur intervention, l'analyse logique s'avère une avenue à considérer et qui mérite d’être plus amplement documentée.

\section{REMERCIEMENTS}

Nous tenons à remercier chaleureusement Mmes Karine Provencher et Myriam Joyal, cliniciennes au CIUSSS du Centre-Sud-de-l'Île-de-Montréal, les deux conceptrices du programme ADOPRO ayant participé au projet, sans qui l'analyse logique du programme n’aurait pu être possible. 


\section{NOTE}

1 Pour une discussion plus approfondie sur les approches théoriques en évaluation en comparaison avec l'analyse logique, voir Tremblay, Brousselle, Richard et Beaudet, 2013.

\section{RÉFÉRENCES}

Blake, S. C. et Ottoson, J. M. (2009). Knowledge utilization: Implications for evaluation. New Directions for Evaluation, 2009(124), 21-34. https://doi.org/10.1002/ev.311

Brousselle, A. et Champagne, F. (2011). Program theory evaluation: Logic analysis. Evaluation and Program Planning, 34(1), 69-78. https://doi.org/10.1016/j.evalprogplan.2010.04.001 Medline:20541262

Chaire CJM-IU-UQAM. (s.d.). Lapplication des connaissances dans le domaine psychosocial. Repéré à https://chairecjmiu.uqam.ca/component/content/article/22/67lapplication-des-connaissances-dans-le-domaine-psychosocialdetail.html

Champagne, F., Brousselle, A., Contandriopoulos, A.-P. et Hartz, Z. (2009). Lanalyse logique. Dans A. Brousselle, F. Champagne, A.-P. Contandriopoulos et Z. Hartz (éds.), Lévaluation: concepts et méthodes (p. 103-112). Montréal: Les Presses de l'Université de Montréal.

CRDITED de Montréal. (2015). ADOPRO : un programme de développement des habiletés sociales pour les adolescents de 12 à 17 ans présentant une déficience intellectuelle. Montréal: CRDITED de Montréal.

Davison, C. M. (2009). Knowledge translation: Implications for evaluation. New Directions for Evaluation, 2009(124), 75-87. https://doi.org/10.1002/ev.315

Donnelly, C., Letts, L., Klinger, D. et Shulha, L. (2014). Supporting knowledge translation through evaluation: evaluator as knowledge broker. Canadian Journal of Program Evaluation, 29(1), 36-61. https://doi.org/10.3138/cjpe.29.1.36

Mayne, J. (2001). Addressing attribution through contribution analysis: Using performance measures sensibly. Canadian Journal of Program Evaluation, 16(1), 1-24.

Ministère de la Santé et des Services sociaux. (2001). De l'intégration sociale à la participation sociale. Politique de soutien aux personnes présentant une déficience intellectuelle, valeurs familles et aux autres proches. Repéré à http://publications.msss.gouv.qc.ca/ msss/fichiers/2001/01-840-02.pdf

Tremblay, M.-C., Brousselle, A., Richard, L. et Beaudet, N. (2013). Defining, illustrating and reflecting on logic analysis with an example from a professional development program. Evaluation and Program Planning, 40, 64-73. https://doi.org/10.1016/j. evalprogplan.2013.05.004 Medline:23807118

World Health Organization (2004). World report on knowledge for better health: strengthening health systems. Repéré à http://www.who.int/rpc/meetings/en/world_report_on_ knowledge_for_better_health2.pdf

\section{PRÉSENTATION DES AUTEURS}

Anne-Marie Ouimet est titulaire d'une maîtrise en santé communautaire de l'Université de Montréal. Elle travaille à titre de professionnelle de recherche au Centre de recherche et 
d'expertise sur la déficience intellectuelle et le trouble du spectre de l'autisme du CIUSSS du Centre-Sud-de-l'Île-de-Montréal. Elle possède une expertise en recherche évaluative et en méthodologie qualitative.

Daphné Morin détient une maîtrise en sociologie de l'Université du Québec à Montréal. Elle est responsable du Centre de recherche et d'expertise sur la déficience intellectuelle et le trouble du spectre de l'autisme du CIUSSS du Centre-Sud-de-l'Île-de-Montréal. Depuis dix ans, elle est engagée dans le développement d'une culture de recherche en milieu de pratique en DI et TSA. Elle a acquis une expérience de plus de 20 ans en recherche sociale, notamment sur les phénomènes d’exclusion sociale. 